\title{
Evaluation of Anxiety, Burnout and Psychological Resilience Levels of Healthcare Workers in the COVID-19 Pandemic
}

${ }^{1}$ University of Health Sciences, Ankara City Hospital, Department of Psychiatry, Ankara, Turkey

Hasan KAYA

Aybeniz CIVAN KAHVE

Saniye Gökçe SAYKAL

Nihan BÜYÜKLÜOĞLU

Rabia Nazik YÜKSEL

Erol GÖKA

Correspondence: Rabia Nazik YÜKSEL University of Health Sciences, Ankara City Hospital, Department of Psychiatry, Ankara, Turkey

Phone: +903125526000

E-mail: rabianazik@gmail.com

\author{
Hasan Kaya ${ }^{1}$ (D) , Aybeniz Civan Kahve' ${ }^{1}$ (D) , Saniye Gökçe Saykal ${ }^{1}$ (iD , \\ Nihan Büyüklüoğlư ${ }^{1}$ (D) , Rabia Nazik Yüksel ${ }^{1}$ (iD) , Erol Göka ${ }^{1}$ (iD
}

\section{ABSTRACT}

Purpose: The COVID-19 pandemic affects mental health as well as physical health. Many studies have shown that the pandemic has negative effects on the mental health of individuals. Determining the factors that play a role in the psychological resilience and burnout levels of healthcare workers, who are at the forefront of the pandemic, will play an important role in preventive and therapeutic planning in pandemic management. Our aim is to evaluate the anxietyburnout levels and psychological resilience levels in healthcare workers and the factors affecting them.

Method:Sociodemographic data form, State-Trait Anxiety Scale (STAI), Connor-Davidson Psychological Resilience Scale (CD-RISC) and Maslach Burnout Inventory (MBI), were created in Google Documents and applied to the Healthcare workers via mail and message groups and social media groups. A total of 257 people were included in the study.

Results: In our study, high trait anxiety scores and increased working were associated with increased levels of burnout. Doctors and nurses showed more signs of burnout than auxiliary assistant staff. The emotional exhaustion and personal accomplishment were higher in females. Psychological resilience could predict personal accomplishment sub-dimension and total scores of MBI.

Conclusions: Healthcare workers are at risk for burnout. Working conditions should be regulated and psychosocial support programs should be established for healthcare workers who are at the forefront in pandemic.

Keywords: COVID-19, mental health, healthcare workers, burnout, resilience
COVID-19 Pandemisinde Sağlık Çalışanlarının Anksiyete, Tükenmişlik ve Psikolojik Dayanıklılık Açısından Değerlendirilmesi

ÖZET

Giriş ve Amaç: COVID-19 pandemisi, hem beden sağlığını hem de ruh sağlığını etkilemektedir. Bir çok çalışmada pandeminin bireylerin ruh sağlığı üzerine olumsuz etkileri olduğu gösterilmiş̧tir. Pandeminin ön saflarında mücadele eden sağlık çalışanlarının psikolojik rezilyansları ve tükenmişlik seviyeleri üzerinde rolü olan etmenleri belirlemek, koruyucu ve terapötik önlemler almak ve pandemiyi yönetmek açısından önemlidir. Bu çalışmada amacımız, sağlık çalışanlarında anksiyete, tükenmişlik ve psikolojik rezilyans seviyelerini ve bunları etkileyen faktörleri değerlendirmektir.

Yöntem ve Gereçler: Google Dökümanlar üzerinden, sosyodemografik veri formu, Durumluk ve Süreklilik Anksiyete Ölçeği, Connor-Davidson Psikolojik Rezilyans ölçeği, Maslach Tükenmişlik Envanterini (MTE) içeren anket oluşturularak sağlık çalışanlarına e-posta, mesaj grupları ve sosyal medya grupları aracılığılla ulaştıııldı. İki yüz elli yedi kişi çalışmaya katıldı.

Bulgular: Çalışmamızda, sürekli kaygı skorlarında yükseklik ve artmış çalışma saatleri, tükenmişlik seviyelerinde artışla ilişkili bulundu. Doktorlar ve hemşireler, yardımcı sağlık personellerine göre daha çok anksiyete ve tükenmişlik belirtisi gösteriyordu. Emosyonel tükenme ve kişisel başarıda düşme, kadınlarda daha yüksekti. Psikolojik rezilyansın, MTE toplam skorlarını ve kişisel başarıda düşme düzeylerini predikte edebileceği bulundu.

Tartışma ve Sonuç: Sağlık çalışanları, tükenmişlik için risk altındadır. Pandemide ön safta çalışan sağık çalışanlarının çalışma şartları düzenlenmeli ve psikososyal destek programları oluşturulmalıdır.

Anahtar Kelimeler: COVID-19, koronavirüs, ruh sağlığı, sağlık çalışanları, tükenmişlik, rezilyans, anksiyete 
$\mathbf{T}$ he new type of coronavirus (SARS-CoV-2), which emerged in Wuhan, China in December 2019, spread rapidly in a short time and affected the whole world. While COVID-19 disease caused by SARS-CoV-2 is seen as an upper respiratory tract infection with asymptomatic or mild symptoms in general, viral pneumonia may cause severe clinical manifestations that progress to acute respiratory failure in some cases. Depending on the clinical condition of the patients, outpatient or inpatient treatment may be required. The rapid spread of the virus, the variety of symptoms, and the absence of a definitive treatment have caused excessive intensity and burden in the health system.

Although the prevalence of COVID-19 among healthcare workers varies in different countries, it constitutes a significant proportion of those affected. According to some reports, $10 \%$ of confirmed COVID-19 cases involved healthcare providers(1). Healthcare workers' burden continues to increase due to the pandemic and the overload in the health system. Healthcare workers found themselves working at the vanguard of responding to the COVID-19 pandemic and exposed to various risks to their psychological and physical health (2).

The fatal and uncontrollable nature of COVID-19, with no known effective cure, is coupled with a relatively high infection rate and mortality among healthcare providers. Separation from the family, the risk of transmitting the virus to family members, mental and physical fatigue caused by excessive working hours, problems in accessing protective equipment and medical supplies, working in a field outside of their specialties have provoked feelings of anxiety and stress on healthcare workers than ever before (3).

Burnout is a psychological condition that is defined as a psychological, emotional, and physical stress state in response to prolonged exposure to occupational stress (4). Maslach et al described three subtitles of burnout as; emotional exhaustion, depersonalization, and decreased professional success (4). Emotional exhaustion represents the primary dimension of personal stress, the depletion of emotional resources required to communicate with other people. Desensitization represents the interpersonal context dimension of burnout. It refers to developing negative feelings and cynical attitudes towards the person to whom the person provides service or care. The individual skills-achievement reduction component is the selfassessment dimension of burnout and refers to feelings of inadequacy and lack of success and productivity at work(5).

Burnout can have severe outcomes for both patients and healthcare workers. It can cause dreadful physical and mental health consequences and reduce the quality of service provided by affected staff. Various systematic reviews in the literature have found that high burnout in healthcare workers is associated with less safe patient care $(6,7)$.

Recognizing the anxiety and burnout levels of healthcare professionals can contribute to planning preventive mental health services and providing mental well-being for healthcare workers. Therefore, we aimed to determine the anxiety and burnout levels in healthcare workers of the COVID-19 pandemic in a pandemic hospital in Turkey and reveal the potential protective and risk factors.

\section{METHODS}

\section{Sample}

This study was conducted between June 1, 2020, and July 1,2020 , by applying an anonymous online questionnaire to people working at Ankara City Hospital during the COVID-19 outbreak. All participants were informed consent electronically before enrollment and were informed that they could withdraw their consent at any time. Those who completed the questionnaires incompletely were excluded from the study. Sociodemographic data form, State-Trait Anxiety Scale (STAI), ConnorDavidson Psychological Resilience Scale (CD-RISC), and Maslach Burnout Inventory (MBI), were created in Google Documents and applied to the healthcare workers via mail and message groups and social media groups. Two hundred fifty-six people agreed to participate in the study and fill out the forms completely.

\section{Measurement tools}

\section{State-Trait Anxiety Inventory (STAI)}

This scale was developed by Spielberger et al. It includes two separate scales measuring trait and situational anxiety levels(8). The first 20 items measure state anxiety, and the second 20 items measure trait anxiety. Turkish validity and reliability study was conducted by Öner and Le Compte (9). 


\section{Connor-Davidson Psychological Resilience Scale (CD-RISC)}

It is a 5-point Likert-type scale with 25 items. It was developed by Connor and Davidson and measures psychological health (10). The higher scores on the scale mean higher psychological resilience. Turkish validity and reliability study was conducted by Karairmak (11).

\section{Maslach Burnout Inventory (MBI)}

Maslach and Jackson developed it to determine the level of burnout (12). This scale consists of 22 items. It has three sub-dimensions: emotional exhaustion, depersonalization, and personal accomplishment. The high score from the emotional exhaustion and depersonalization subdimensions and the low score from the personal accomplishment sub-dimension indicate the level of burnout. The Turkish validity and reliability study was conducted by Ergin (13). However, while using the "personal accomplishment" subscale in analysis and determining the total scores, the scores were calculated by reverse coding the answers given to the items. Thus, it was ensured that high scores in this subscale showed high burnout in those who received high scores.

\section{Statistical Analysis}

SPSS 22 program was used to analyze the data. For the distribution of normality, histogram, Skewness, and Kurtosis values were used in addition to the KolmogorovSmirnov test. The chi-square test was used to compare categorical variables, Pearson correlation was used for correlation analysis of values with a normal distribution, and Spearman correlation analysis was performed for those not showing normal distribution. Independent Samples T-Test was used for comparing the means of two independent groups showing normal distribution, and the Mann Whitney $\mathrm{U}$ test was used to compare the medians of two independent groups that did not show normal distribution. One Way ANOVA test was used for comparisons of 3 independent groups showing normal distribution. In comparing three independent groups, if the variants' distribution is not homogeneous (Levene's $p<, 05$ ), Welch statistics were used. The Kruskal Wallis $\mathrm{H}$ test was used for comparing three independent groups of scales that did not show normal distribution. In cases where there was a significant difference between the groups, two-group comparisons were made, and Bonferroni correction was applied to determine which groups the difference was between them. Stepwise multiple linear regression analysis was performed to determine potential risk and protective factors for burnout. The significance level was accepted as $p<0.05$.

\section{RESULTS}

257 people (mean age: $38.84 \pm 8.53$ ) participated in this study. $83.3 \%$ (n: 214 ) of the participants were female and $16.7 \%$ (n: 43) were male. Of the participants, $26.1 \%$ (n: 67 ) were doctors, $56.0 \%$ (n: 144) nurses, $17.9 \%$ (n: 46) health staff. Forty-seven point five percent ( $\mathrm{n}: 122)$ were married, $52.5 \%$ ( $\mathrm{n}: 135$ ) were single / widowed. Sixty-one point five percent (n: 158) did not have children, 38.5\% (n: 99) have children and $66.9 \%$ (n: 172) live with their family or friends, $33.1 \%$ (n: 85) live alone. While $49.4 \%$ (n: 127) people did not live with people at risk for COVID-19, 50.6\% (n: 130) people were living with people at risk for COVID-19. The socio-demographic information of the sample is shown in Table 1.

The mean year of education was $15.02 \pm 1.50$. The participants' mean working period was $113.95 \pm 111.83$ months (min: 6, max: 456 months). Twenty-eight point four percent ( $n: 73$ ) of the participants had a known chronic disease. Eighteen point three percent ( $n: 43$ ) declared that they had psychiatric diagnosis and treatment before. While $48.2 \%$ (n: 124 ) of the participants worked in the COVID-19 service, $45.9 \%$ (n: 118) stated that their weekly working hours increased during the pandemic.

The differences between the CD-RISC, STAI-T, STAI-S, and total $\mathrm{MBI}$ score medians (IQR) of the healthcare workers are given in Table 2.

The emotional exhaustion median scores of the healthcare workers were higher in females (25.5 [13.3] vs 20 [15]; z $=-2.885 ; \mathrm{P}=0.004)$, working in COVID-19 clinics (26[11] vs 24 [15]; $z=-2,576 ; P=0,01)$, who had excessive weekly working hours $(26[10,3]$ vs $23[12] ; z=-3,492 ; P<0,001)$, and who had a psychiatric diagnosis before (27[12] vs 25[12]; $\mathrm{z}=-2,040 ; \mathrm{P}=0,041)$. Emotional exhausting median scores were 26 (9), 26 (14.8) and 17 (12) in doctor, nurse and assistant staff, respectively. ( $\left.\mathrm{x}^{2}: 30,776 ; \mathrm{P}<0.001\right)$ Median score of doctors $(z=-4.706 ; P<0.001)$ and nurses $(z=-5,314 ; P$ $<0.001)$ were statistically higher than assistant staff. 


\begin{tabular}{|c|c|c|}
\hline & $\mathrm{N}$ & $\%$ \\
\hline \multicolumn{3}{|l|}{ Gender } \\
\hline Female & 214 & 83.3 \\
\hline Male & 43 & 16.7 \\
\hline \multicolumn{3}{|l|}{ Marital status } \\
\hline Married & 122 & 47.5 \\
\hline Single & 135 & 52.5 \\
\hline \multicolumn{3}{|l|}{ Professional status } \\
\hline Doctor & 67 & 26.1 \\
\hline Nurse & 144 & 56.0 \\
\hline Assistant staff & 46 & 17.9 \\
\hline \multicolumn{3}{|l|}{ Having children } \\
\hline Yes & 99 & 38.5 \\
\hline No & 158 & 61.5 \\
\hline \multicolumn{3}{|l|}{ Living condition } \\
\hline Alone & 85 & 33.1 \\
\hline With friends or family & 172 & 66.9 \\
\hline
\end{tabular}

Depersonalization median scores of the participants were higher in those who were not married (11 [5] vs 9 [5]; $z=$ -2.616; $P=0.009$ ), did not have children (11 [4] vs 8 [4]; $z=-4,445 ; P<0.001)$, worked in the COVID-19 clinics (11 [6] vs $10[5] ; z=-2.656 ; P=0.008)$, and had excessive weekly working hours (11 [5.3] vs 9 [5]; $z=-4,065 ; P<0.001)$. Depersonalization median scores were 11 (5), 10 (4) and 8 (6) in doctor, nurse, and assistant staff, respectively. $\left(x^{2}\right.$ : $10,825 ; \mathrm{P}=0.004)$ Depersonalization median score of doctors was statistically higher than assistant staff. $(z=-3,189$; $P=0.001)$.

Personal accomplishment median scores of the participants were higher in females (21 [6] vs 19 [7]; $z=-2.0366 ; P$ $=0.042$ ), worked in the COVID-19 clinics (21 [5] vs 20 [8]; $z$ $=-2.342 ; \mathrm{P}=0.019)$ and and who had a psychiatric diagnosis before (22[6] vs 20[7]; $z=-1,994 ; P=0,046)$. Personal accomplishment median scores were 22 (7), 20 (7) and 19,5 $(7,25)$ in doctor, nurse, and assistant staff, respectively. $\left(x^{2}\right.$ : 7,096; $\mathrm{P}=0.029$ ). Personal accomplishment median score of doctors was statistically higher than assistant staff. ( $\mathrm{z}=$ $-2,629 ; P=0.009$ ).

According to the participants' sociodemographic and working characteristics, the comparison of CD-RISC, STAI-T, STAI-S, and total scores of MBlis shown in Table 2.
A correlation analysis between sociodemographic variables, study characteristics, CD-RISC, STAI-T, STAI-S, MBI's subscales (emotional exhaustion, personal accomplishment, depersonalization), and total scores is shown in Table 3.

In the multiple regression analysis to predict the subscale and total scores of $\mathrm{MBI}$, important regression equations were found for the MBI total $(F(1,250)=60,495$; Adjusted $R 2: 0,538 ; p<0.001)$, the Emotional exhaustion $(F(1.251)=$ 54.722; Adjusted R2: 0.457; $p<0.001)$, Depersonalization $(F(1.251)=22.718$; Adjusted R2: 0.254; $p<0.001)$, and personal accomplishment $(F(1,252)=51,945$; Adjusted R2: $0.375 ; \mathrm{p}<0.001)$, and total MBI. These models are shown in Table 4.

\section{DISCUSSION}

In this cross-sectional study, we evaluated healthcare workers' burnout levels in the COVID-19 pandemic and revealed the factors that may affect burnout. We found that higher trait anxiety, working as a nurse or doctor, increased years of education and excessive weekly working hours are associated with increased burnout levels.

COVID-19 pandemic impacts the physical and psychological health of healthcare workers worldwide (14). Not surprisingly, it creates unprecedented challenges for healthcare professionals. Previous research on burnout has shown that the highest burnout rate is observed among hospital emergency departments (15). In the event of a pandemic that caused a crisis in healthcare provision, all healthcare workers tried to overcome this crisis by acting in collaboration. However, as the pandemic period extends, the burnout of healthcare workers is expected to increase.

Our findings reinforce the multidimensionality of burnout. The total and three dimensions of burnout are related to a set of variables. It can be thought that these three sub-dimensions may be necessary for terms of considering the future burnout prevention program.

We have determined that female gender, marital status, parental status, working status in the COVID-19 clinics, excessive working hours, and living alone may contribute to burnout. 
Table 2: Comparison of CD-RISC, STAI-T, STAI-S, and MBI scores according to sociodemographic variables and working conditions

\begin{tabular}{|c|c|c|c|c|c|c|c|c|}
\hline & $\begin{array}{l}\text { CD-RISC } \\
\text { (Median,IQR*) }\end{array}$ & $\begin{array}{l}\text { Statistical } \\
\text { value }\end{array}$ & $\begin{array}{l}\text { STAI-T } \\
\text { (Median,IQR*) }\end{array}$ & $\begin{array}{l}\text { Statistical } \\
\text { value }\end{array}$ & $\begin{array}{l}\text { STAI-S } \\
\text { (Median,IQR*) }\end{array}$ & $\begin{array}{l}\text { Statistical } \\
\text { value }\end{array}$ & $\begin{array}{l}\text { Bornout } \\
\text { (Median,IQR*) }\end{array}$ & $\begin{array}{l}\text { Statistical } \\
\text { value** }^{* *}\end{array}$ \\
\hline \multicolumn{9}{|l|}{ Gender } \\
\hline Female & $81,5(22)$ & \multirow{2}{*}{$\begin{array}{l}Z:-3,823 \\
p<0.001\end{array}$} & $40(11)$ & \multirow{2}{*}{$\begin{array}{l}\text { Z:-3,554 } \\
p<0.001\end{array}$} & $41(14)$ & \multirow{2}{*}{$\begin{array}{l}Z:-4,042 \\
p<0.001\end{array}$} & $57(19)$ & \multirow{2}{*}{$\begin{array}{l}\text { Z:-2,995 } \\
\mathrm{p}: 0,003\end{array}$} \\
\hline Male & $96,0(67)$ & & $35(13)$ & & $31(15)$ & & $50(25)$ & \\
\hline \multicolumn{9}{|c|}{ Marital status } \\
\hline Married & $84,5(25.5)$ & \multirow{2}{*}{$\begin{array}{l}Z:-1,808 \\
\text { p:0.071 }\end{array}$} & $38,5(12)$ & \multirow{2}{*}{$\begin{array}{l}\text { Z:-1,399 } \\
\text { p: } 0.162\end{array}$} & $40(16.3)$ & \multirow{2}{*}{$\begin{array}{l}\text { Z:-0,614 } \\
\text { p:0,539 }\end{array}$} & $53,5(19)$ & \multirow{2}{*}{$\begin{array}{l}\text { Z:-1,871 } \\
\text { p:0,061 }\end{array}$} \\
\hline Single & $81(23)$ & & $40(13)$ & & $41(15)$ & & $57(21)$ & \\
\hline \multicolumn{9}{|c|}{ Professional status } \\
\hline Doctor & $83(22)^{a}$ & \multirow{3}{*}{$\begin{array}{l}x^{2}: 22,162 \\
P<0.001\end{array}$} & $39(10)$ & \multirow{3}{*}{$\begin{array}{l}x^{2}: 12,910 \\
P<0.001\end{array}$} & $40(14)$ & \multirow{3}{*}{$\begin{array}{l}x^{2}: 17,312 \\
P<0.001\end{array}$} & $60(15) a$ & \multirow{3}{*}{$\begin{array}{l}x^{2}: 26,596 \\
p<0.001\end{array}$} \\
\hline Nurse & $79(23)^{a}$ & & $41(12) a$ & & $42(13,8) a$ & & $57(23,5) a$ & \\
\hline Health staff & $92,5(27)$ & & $34,5(16,3)$ & & $31,5(21)$ & & $46(14,5)$ & \\
\hline \multicolumn{9}{|c|}{ Having children } \\
\hline Yes & $84(25)$ & \multirow{2}{*}{$\begin{array}{l}\text { Z:-0,883 } \\
\text { p:0.377 }\end{array}$} & $38(12)$ & \multirow{2}{*}{$\begin{array}{l}\text { Z:-1,405 } \\
\text { p:0,166 }\end{array}$} & $40(17)$ & \multirow{2}{*}{$\begin{array}{l}\text { Z:-0,738 } \\
\text { p:0.460 }\end{array}$} & $52(18)$ & \multirow{2}{*}{$\begin{array}{l}\text { Z:-2,443 } \\
\text { p:0.013 }\end{array}$} \\
\hline No & $82,5(21,3)$ & & $40(12,3)$ & & $41(15)$ & & $57,5(16)$ & \\
\hline \multicolumn{9}{|c|}{ Living conditions } \\
\hline $\begin{array}{l}\text { lived with } \\
\text { friend or } \\
\text { family }\end{array}$ & $84,5(23)$ & \multirow[t]{2}{*}{$\begin{array}{l}\text { Z:-2,300 } \\
\text { p:0.021 }\end{array}$} & $39(11,8)$ & \multirow[t]{2}{*}{$\begin{array}{l}\text { Z:-2,080 } \\
\text { p:0.038 }\end{array}$} & $40(15,8)$ & \multirow[t]{2}{*}{$\begin{array}{l}\text { Z:-1,214 } \\
\text { p:0,225 }\end{array}$} & $54,5(19,75$ & \multirow[t]{2}{*}{$\begin{array}{l}\text { Z:-1,942 } \\
\text { p:0.052 }\end{array}$} \\
\hline Alone & $78(26,5)$ & & $42(12)$ & & $41(15)$ & & $58(23,5)$ & \\
\hline \multicolumn{9}{|c|}{ Having a psychiatric diagnosis in the past } \\
\hline Yes & $72(19)$ & \multirow{2}{*}{$\begin{array}{l}Z:-3,571 \\
P<0.001\end{array}$} & $45(10)$ & \multirow{2}{*}{$\begin{array}{l}Z:-4,088 \\
p<0.001\end{array}$} & $47(13)$ & \multirow{2}{*}{$\begin{array}{l}Z:-3,825 \\
p<0.001\end{array}$} & $60(27)$ & \multirow{2}{*}{$\begin{array}{l}\text { Z:-2,191 } \\
\text { p:0.028 }\end{array}$} \\
\hline No & $85(26)$ & & $38(12)$ & & $39(15)$ & & $55(20,25)$ & \\
\hline \multicolumn{9}{|c|}{ Working in the COVID-19 clinics } \\
\hline Yes & $81(19,8)$ & \multirow{2}{*}{$\begin{array}{l}Z:-1,473 \\
\text { p: } 0,141\end{array}$} & $40(11,6)$ & Z:-1,811 & $42(14)$ & Z:-1,664 & $59,5(17,5)$ & $Z:-3,295$ \\
\hline No & $85(28,5$ & & $39(12)$ & p:0.0/0 & $39(16)$ & p:0,090 & $52(20)$ & p:0,001 \\
\hline Weekly work & g hours increase & & & & & & & \\
\hline Yes & $81(22)$ & Z:-1,673 & $40(12)$ & $Z:-1,949$ & $43(17)$ & $Z:-2,924$ & $60(17)$ & Z:-3,944 \\
\hline No & $86(25)$ & p:0,094 & $38(12)$ & p:0,051 & $39(15)$ & p:0,003 & $52(19)$ & $p<0.001$ \\
\hline
\end{tabular}




\section{Table 3: Correlation between burnout, anxiety, resilience levels with working conditions and socio-demographic variablest}

\begin{tabular}{|c|c|c|c|c|c|c|c|c|c|c|c|c|c|c|c|c|c|}
\hline & 1 & 2 & 3 & 4 & 5 & 6 & 7 & 8 & 9 & 10 & 11 & 12 & 13 & 14 & 15 & 16 & 17 \\
\hline$\therefore \stackrel{8}{8}$ & - & & & & & & & & & & & & & & & & \\
\hline 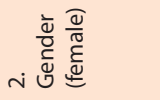 & 0,057 & - & & & & & & & & & & & & & & & \\
\hline 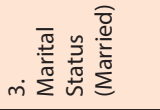 & $\begin{array}{c}- \\
, 514^{* *}\end{array}$ & $-0,054$ & - & & & & & & & & & & & & & & \\
\hline 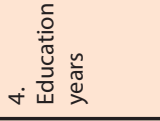 & 0,047 & 0,027 & 0,01 & $\begin{array}{ll}- & - \\
-1\end{array}$ & & & & & & & & & & & & & \\
\hline 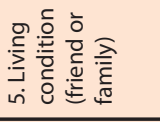 & $321^{* *}$ & $-0,005$ &, $387^{* *}$ & 0,081 & - & & & & & & & & & & & & \\
\hline 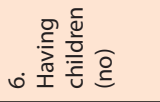 & ,646** & 0,009 & $\begin{array}{c}- \\
, 689^{* *}\end{array}$ & $-0,05$ & $\begin{array}{c}- \\
, 302^{* *}\end{array}$ & - & & & & & & & & & & & \\
\hline 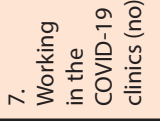 & $\begin{array}{c}- \\
, 247^{* *}\end{array}$ & $-0,036$ & 0,107 &, $131^{*}$ &, $215^{* *}$ & $-0,076$ & - & & & & & & & & & & \\
\hline 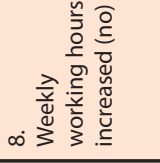 & , $328^{* *}$ & 0,005 & , $188^{* *}$ & 0,042 & $182^{* *}$ & $\begin{array}{c}- \\
248^{* *}\end{array}$ &, $189^{* *}$ & - & & & & & & & & & \\
\hline 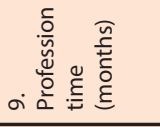 & $894^{* *}$ & $-0,033$ & $\begin{array}{c}- \\
, 525^{* *}\end{array}$ & $-0,005$ & $\begin{array}{c}- \\
, 379^{* *}\end{array}$ &, $674^{* *}$ &, $162^{* *}$ &, $352^{-}$ & - & & & & & & & & \\
\hline 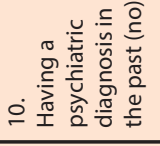 &, $184^{* *}$ & 0,058 & $-0,034$ & $-0,038$ & $-0,033$ & 0,101 & 0,007 & $-0,052$ & 0,097 & - & & & & & & & \\
\hline 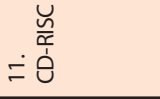 & 0,077 & ,239** & $-0,113$ & 0,005 & $-144^{*}$ & 0,055 & $-0,092$ & $-0,105$ & 0,051 & $\overline{-}^{-} 223^{* *}$ & - & & & & & & \\
\hline$\stackrel{\frac{1}{5}}{\frac{1}{5}}$ &,$- 138^{*}$ & ${ }^{-} 222^{* *}$ & 0,087 & 0,003 &, $130^{*}$ & $-0,088$ & 0,113 & 0,122 & $-0,086$ &, $255^{* *}$ & $\begin{array}{c}- \\
, 718^{* *}\end{array}$ & - & & & & & \\
\hline$m \stackrel{\frac{n}{k}}{m}$ & $-0,083$ & $\begin{array}{c}- \\
, 253^{* *}\end{array}$ & 0,038 & 0,052 & 0,076 & $-0,046$ & 0,104 &, $183^{* *}$ & $-0,054$ &, $239 * *$ & $\begin{array}{c}- \\
, 665^{* *}\end{array}$ & ,746 & - & & & & \\
\hline 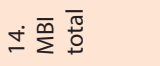 &,$- 161^{*}$ & $\begin{array}{c}- \\
187^{* *}\end{array}$ & 0,117 & ,189** & 0,121 & $-153^{*}$ & $206 * *$ &, $247^{* *}$ & $-0,107$ & ,137* & $\begin{array}{c}- \\
, 589^{* *}\end{array}$ &, $666^{* *}$ & ,610** & - & & & \\
\hline 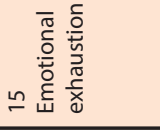 & $-0,092$ & , $180^{* *}$ & 0,09 & $200 * *$ & 0,044 & $-0,103$ &, $161^{* *}$ &, $218^{* *}$ & $-0,034$ & ,128* & $\begin{array}{c}- \\
, 493^{* *}\end{array}$ & ,598** &, $594 * *$ &, $900 * *$ & - & & \\
\hline 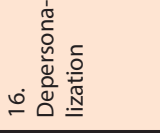 & $\stackrel{-}{-250^{* *}}$ & $-0,098$ &, $163^{* *}$ &, $131^{*}$ & 0,115 & $\begin{array}{c}- \\
, 278^{* *}\end{array}$ &, $166^{* *}$ &, $254^{* *}$ & $\begin{array}{c}- \\
180^{* *}\end{array}$ & 0,058 & $\stackrel{-}{-} 279^{* *}$ & $434^{* *}$ &, $282^{* *}$ &, $681 * *$ &, $524^{* *}$ & - & \\
\hline 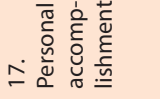 & $-0,101$ &,$- 127^{*}$ & 0,047 & 0,084 & ,151* & $-0,012$ & , 146* & 0,115 & $-0,086$ & ,125* & $\stackrel{-}{-567^{* *}}$ &, $483^{* *}$ &, $437^{* *}$ &, $647^{* *}$ &, $349 * *$ &, $269 * *$ & - \\
\hline
\end{tabular}


Table 4: Multiple regression models of sub-dimensions and total scores of Maslach Burnout Inventory (MBI)

\begin{tabular}{|c|c|c|c|c|c|c|c|c|c|c|}
\hline \multirow[b]{2}{*}{$\begin{array}{l}\text { Emotional } \\
\text { exhaustion }\end{array}$} & \multirow{2}{*}{$\begin{array}{l}\text { Predictors } \\
\text { (Constant) }\end{array}$} & \multirow{2}{*}{$\begin{array}{c}\text { Regression } \\
\text { coefficient } \\
(\beta)\end{array}$} & \multirow{2}{*}{$\begin{array}{c}\text { S.E. } \\
4,505\end{array}$} & \multirow[t]{2}{*}{$\begin{array}{l}\text { Standardized } \\
\text { re-gression } \\
\text { coeffi-cient } \\
\text { (Beta) }\end{array}$} & \multirow{2}{*}{$\frac{t}{-2,405}$} & \multirow{2}{*}{$\begin{array}{c}p \\
0,017\end{array}$} & \multicolumn{2}{|c|}{$\begin{array}{l}95 \% \text { confidence interval } \\
\text { of } \beta\end{array}$} & \multirow[t]{2}{*}{ Tolerance } & \multirow[t]{2}{*}{ VIF } \\
\hline & & & & & & & $-19,71$ & $-1,963$ & & \\
\hline & STAI-T & 0,367 & 0,071 & 0,368 & 5,148 & $<0,001$ & 0,226 & 0,507 & 0,416 & 2,401 \\
\hline & $\begin{array}{l}\text { Doctor or } \\
\text { nurse }\end{array}$ & 3,93 & 1,153 & 0,172 & 3,408 & 0,001 & 1,659 & 6,202 & 0,834 & 1,199 \\
\hline & STAI-S & 0,215 & 0,059 & 0,265 & 3,648 & $<0,001$ & 0,099 & 0,331 & 0,404 & 2,474 \\
\hline & $\begin{array}{l}\text { Education } \\
\text { years }\end{array}$ & 0,633 & 0,285 & 0,109 & 2,219 & 0,027 & 0,071 & 1,195 & 0,888 & 1,126 \\
\hline \multirow[t]{5}{*}{ Depersonalization } & (Constant) & 4,592 & 1,198 & & 3,832 & $<0,001$ & 2,232 & 6,952 & & \\
\hline & STAI-T & 0,178 & 0,023 & 0,416 & 7,587 & $<0,001$ & 0,132 & 0,224 & 0,975 & 1,026 \\
\hline & $\begin{array}{l}\text { Having } \\
\text { children }\end{array}$ & $-1,36$ & 0,436 & $-0,175$ & $-3,122$ & 0,002 & $-2,217$ & $-0,502$ & 0,927 & 1,079 \\
\hline & Doctor & 1,027 & 0,475 & 0,12 & 2,164 & 0,031 & 0,093 & 1,962 & 0,955 & 1,048 \\
\hline & $\begin{array}{l}\text { Weekly } \\
\text { working } \\
\text { hours } \\
\text { increased }\end{array}$ & 0,851 & 0,428 & 0,113 & 1,987 & 0,048 & 0,008 & 1,695 & 0,911 & 1,098 \\
\hline \multirow[t]{4}{*}{$\begin{array}{c}\text { Personal } \\
\text { accomplishment }\end{array}$} & (Constant) & 26,845 & 3,111 & & 8,629 & $<0,001$ & 20,718 & 32,972 & & \\
\hline & CD-RISC & $-0,134$ & 0,021 & $-0,458$ & $-6,459$ & $<0,001$ & $-0,175$ & $-0,093$ & 0,488 & 2,05 \\
\hline & Doctor & 1,739 & 0,581 & 0,149 & 2,992 & 0,003 & 0,594 & 2,884 & 0,993 & 1,007 \\
\hline & STAI-T & 0,108 & 0,042 & 0,185 & 2,609 & 0,01 & 0,027 & 0,19 & 0,486 & 2,059 \\
\hline \multirow[t]{6}{*}{ MBI Total } & (Constant) & 15,739 & 9,553 & & 1,647 & 0,101 & $-3,076$ & 34,555 & & \\
\hline & STAI-T & 0,815 & 0,097 & 0,512 & 8,381 & $<0,001$ & 0,623 & 1,006 & 0,485 & 2,062 \\
\hline & $\begin{array}{l}\text { Doctor or } \\
\text { nurse }\end{array}$ & 3,645 & 1,746 & 0,1 & 2,087 & 0,038 & 0,206 & 7,085 & 0,789 & 1,268 \\
\hline & $\begin{array}{l}\text { Weekly } \\
\text { working } \\
\text { hours } \\
\text { increased }\end{array}$ & 3,690 & 1,227 & 0,131 & 3,007 & 0,003 & 1,273 & 6,108 & 0,947 & 1,056 \\
\hline & $\begin{array}{l}\text { Education } \\
\text { years }\end{array}$ & 1,239 & 0,421 & 0,133 & 2,944 & 0,004 & 0,41 & 2,067 & 0,885 & 1,13 \\
\hline & CD-RISC & $-0,145$ & 0,05 & $-0,181$ & $-2,908$ & 0,004 & $-0,242$ & $-0,047$ & 0,468 & 2,138 \\
\hline
\end{tabular}

Although there were findings in previous studies that being married and having a child could increase burnout $(16,17)$, Burnout scores were lower in those married and had children in our study. This can be explained by reducing the workload outside the hospital with the spouse's support in married couples. It has been shown that having a child significantly reduces the depersonalization subscale values in our study. By increasing the healthcare worker's empathy, having children decreases the depersonalization towards the patients in business life. Also, total burnout scores of those living alone were found to be higher. It can be thought that with social isolation, the loneliness of the healthcare workers can increase burnout.
Our study showed that working in the COVID-19 clinics increased all three dimensions of burnout. In a study conducted in China, burnout prevalence was high among frontline nurses (18). Another study in Switzerland showed a higher level of burnout in the group of healthcare workers who have direct contact with patients (19). In addition to the increase in healthcare workers' workload, the risk of being infected, increasing number of patients, increased disease severity, uncertainty in treatments, critical decision-making processes, the possibility of carrying the pathogen to their family was expected to increase burnout of healthcare workers. 
A previous study conducted with healthcare professionals in Italy showed that weekly working hours predicted three MBI sub-dimensions (20). In our study, both state anxiety and total burnout scores were higher in the group with excessive weekly working hours. Physical and mental fatigue and excessive working hours increased their anxiety and ultimately increased emotional exhaustion and depersonalization sub-dimensions.

A significant positive correlation was found in our study between the duration of education (years) and the emotionally exhausting and depersonalization sub-dimension. It has also been shown that it can predict emotional exhausting sub-dimension and total MBI score. As the years of education increase, the role and responsibilities of individuals in critical decision-making processes increase. It can be thought that this may contribute to mental fatigue and burnout.

When we examined the burnout sub-dimensions and total scores according to professional status in our study, we found that doctors were affected more than assistant staff in the depersonalization and personal accomplishment sub-dimensions. In terms of emotionally exhausting and total $\mathrm{MBI}$ score, we found that doctors and nurses were affected more than assistant staff. In addition to previous studies showing that nurses are at higher risk for burnout $(20,21)$, in a study by Jalili et al., residents are at higher risk than nurses (23). Working as a doctor was found as a predictor in the depersonalization and personal accomplishment sub-dimensions in our study. Here, it can be thought that the uncertainty in COVID-19 treatment and the variability in response to treatment and prognosis may be compelling.

Resilience is defined as the power to cope with risk, trouble, and stress, even though an individual is subjected to a severe strain that can lead to various physical, behavioral, cognitive, and emotional symptoms $(24,25)$. Studies have shown that there tend to be lower resistance rates to infectious disease outbreaks (26). It also suggests that those affected by deterrent events may, over time, overcome adversity and become resilient (27). The traumatic experience created by the pandemic is continuing on society and healthcare workers. In our study, we showed that resilience could predict personal accomplishment sub-dimension and total scores of MBI. We consider it a potentially protective factor against burnout, as shown in previous studies $(16,28)$.
While anxiety can be protective for people at a certain level and time, it becomes harmful by disrupting functionality as its severity and duration increase. COVID-19 pandemic period can be assumed as an example of this. The uncertainty in the treatment and the pandemic's prolongation laid the groundwork for the potentially harmful effects of anxiety.

Limited data are available regarding the mental consequences of the COVID-19 pandemic on healthcare workers with a history of psychiatric disorders. In a study conducted in Australia, it has been shown that those with psychiatric disorders in the past are associated with anxiety, depression, and burnout (29). In our study, consistent with the above study, the STAI-T, STAI-S, and total MBI scores were higher in the group with a history of psychiatric disorders.

According to Spielberger, anxiety is divided into trait anxiety and state anxiety. Trait anxiety is the sensitivity of a person to anxiety, that is, the level of perceiving stressful situations as threats, and state anxiety is the response given after being regarded as threatening to a situation (30).

In our study, trait anxiety-related to anxiety sensitivity was higher in women, individuals living alone, nurses (according to health staff), and those with a history of psychiatric disorders. It was also shown in the regression analysis that it could predict total MBI and its three subdimensions. State anxiety was higher in women, nurses (according to health staff), those with a history of psychiatric disorders, and those whose weekly working hours increased. It has been shown that state anxiety predicts emotional exhaustion.

Individuals with high trait anxiety levels are more likely to experience state anxiety than those with low trait anxiety (in terms of intensity, frequency, duration) (31). In our study, there is a positive high-degree correlation between the trait and state anxiety. We can say that trait anxiety, which is related to the level of anxiety sensitivity determined by biological, environmental, and other factors, is more critical for burnout than state anxiety. However, the continuation of the pandemic and the prolongation of uncertainty causes the anxiety levels to continue increasing. As a result, the emergence of dysfunction and psychiatric disorders will be inevitable.

\section{CONCLUSIONS}

In conclusion, many studies have been conducted on burnout in COVID-19. However, our study is the first to 
examine which subcomponents play a role in burnout and their relationship with resilience.

Our study has some limitations. Firstly, the participants were evaluated cross-sectionally. In hospitals, depending on the pandemic pace, patient density and workload are different. This can affect burnout levels and resilience. In future studies, longer follow-ups evaluating the relationship between state anxiety changes and burnout and resilience can be planned. Secondly, in our study, people who are not interested in internet applications may have been excluded from the sample because the surveys were conducted online. However, in the COVID-19 pandemic, many studies have been conducted online, as face-to-face interviews are risky for the infection.

\section{Ethical considerations}

Ethical approval was obtained from the Ethics Committee of the Ankara City Hospital, Ankara, Turkey with protocol number: E1-20-701. The study was conducted according to the criteria set by the declaration of Helsinki.

\section{REFERENCES}

1. Hassanian-Moghaddam H, Zamani N, Kolahi A-AJCC. COVID-19 pandemic, healthcare providers' contamination, and death: an international view.Crit Care. 2020;24:1-2. https://doi.org/10.1186/ s13054-020-02938-y

2. Lima CKT, de Medeiros Carvalho PM, Lima IDAAS, de Oliveira Nunes JVA, Saraiva JS, de Souza RI. The emotional impact of Coronavirus 2019-nCoV (new Coronavirus disease). Psychiatry Res. 2020; 287, 112915. https://doi.org/10.1007/s40200-020-00643-9

3. Guo J, Liao L, Wang B, Li X, Guo L, Tong Z, et al. Psychological effects of COVID-19 on hospital staff: a national cross-sectional survey of China mainland, Available at SSRN 3550050, 2020. http://dx.doi. org/10.2139/ssrn.3550050

4. Maslach C, Jackson SE, Leiter MP. Maslach burnout inventory manual. Mountain View. CA: Consulting Psychologists Press; 1996.

5. Maslach C, Schaufeli W, Leiter M. Job Burnout. Annu Rev Psychol. 2001; 52, 397-422

6. Dewa CS, Loong D, Bonato S, Trojanowski L. The relationship between physician burnout and quality of healthcare in terms of safety and acceptability: a systematic review. BMJ Open. 2017;7:6, e015141. http://dx.doi.org/10.1136/bmjopen-2016-015141

7. Hall LH, Johnson J, Watt I, TsipaA, O'ConnorDB. Healthcare staff wellbeing, burnout, and patient safety: a systematic review. PloS One. 2016; 11:7, e0159015. https://doi.org/10.1371/journal. pone. 0159015

8. Spielberg C, Gorsuch R, Lushene R. Manual for the State-Trait Anxiety Inventory. Consulting Psychologist Press. Palo Alto. California; 1970.

9. Öner N, Le Compte Va. State-trait anxiety inventory handbook. Bogazici University, Istanbul, Turkey;1985.

10. Connor KM, Davidson JR. Development of a new resilience scale: The Connor-Davidson resilience scale (CD-RISC). Depress Anxiety. 2003;18:76-82. https://doi.org/10.1002/da.10113
11. Karaırmak Ö. Establishing the psychometric qualities of the Connor-Davidson Resilience Scale (CD-RISC) using exploratory and confirmatory factor analysis in a trauma survivor sample. Psychiatry Res. 2010;179:350-356. https://doi.org/10.1016/j. psychres.2009.09.012.

12. Maslach C, Jackson SE. The measurement of experienced burnout. J Organ Behav. 1981;2:99-113. https://doi.org/10.1002/ job.4030020205.

13. Ergin C. Adaptation of burnout and maslach burnout scale in doctors and nurses. in 7 National Congress of Psychology, Association of Turkish Psychologists, Ankara1992. pp. 143-154.

14. Restauri N, Sheridan, AD. Burnout and posttraumatic stress disorder in the coronavirus disease 2019 (COVID-19) pandemic: intersection, impact, and interventions. J Am Coll Radiol. 2020;17(7), 921-926. https://doi.org/10.1016/j.jacr.2020.05.021

15. Chover-Sierra E, Martínez-Sabater A, Lapeña-Moñux Y. Knowledge in palliative care of nursing professionals at a Spanish hospital. Rev Lat Am Enfermagem.2017;25. https://doi. org/10.1590/1518-8345.1610.2847

16. Duarte I, Teixeira A, Castro L, Marina S, Ribeiro C, Jácome C, et al. Burnout among Portuguese healthcare workers during the COVID-19 pandemic. BMC Public Health,2020; 20: 1-10. https://doi. org/10.1186/s12889-020-09980-z

17. Okwaraji F, Aguwa EJP. Burnout, Psychological Distress, and Job Satisfaction among Secondary School Teachers in Enugu, South East Nigeria. J Psychiatry. 2015; 18: 198. http://dx.doi.org/10.4172/ Psychiatry.1000198

18. Hu D, Kong Y, LiW, HanQ, Zhang X, Zhu LX, et al. Frontline nurses' burnout, anxiety, depression, and fear statuses and their associated factors during the COVID-19 outbreak in Wuhan, China: A largescale cross-sectional study. EClinicalMedicine. 2020; 24, 100424 https://doi.org/10.1016/j.eclinm.2020.100424

19. Weilenmann S, Ernst J, Petry H, Sazpinar O, Pfaltz MC, Gehrke S, et al. Health Care WorkersMental Health During the First Weeks of the SARS-CoV-2 Pandemic in Switzerland: A Cross-Sectional Study. medRxiv. 2020. https://doi.org/10.1101/2020.05.04.20088625

20. Giusti EM, Pedroli E, D'Aniello GE, Badiale CS, Pietrabissa G, Manna C, et al. The psychological impact of the COVID-19 outbreak on health professionals: a cross-sectional study. Front Psychol.2020;11. https://doi.org/10.3389/fpsyg.2020.01684

21. Lai J, Ma S, Wang Y, Cai Z, Hu J, Wei N, et al. Factors associated with mental health outcomes among health care workers exposed to coronavirus disease 2019.JAMA Netw Open. 2020;3:e203976-e203976. https://doi:10.1001/ jamanetworkopen.2020.3976

22. Tam CW, Pang EP, Lam LC, Chiu HFJPm. Severe acute respiratory syndrome (SARS) in Hong Kong in 2003: stress and psychological impact among frontline healthcare workers. Psychol Med.2004;34:1197. https://doi.org/10.1017/S0033291704002247

23. Jalili $M$, Niroomand $M$, Hadavand F, Zeinali $K$, Fotouhi AJm. Burnout among healthcare professionals during COVID-19 pandemic: a cross-sectional study. medRxiv.2020. https://doi. org/10.1101/2020.06.12.20129650

24. Cutter SLJNh. The landscape of disaster resilience indicators in the USA. Nat Hazards.2016;80:741-758. https://doi.org/10.1007/ s11069-015-1993-2

25. Ferreira RJ, Cannon C, Buttell FJS. COVID-19: immediate predictors of individual resilience. Sustainability.2020;12:6495. https://doi. org/10.3390/su12166495

26. Bonanno GAJAp. Loss, trauma, and human resilience: Have we underestimated the human capacity to thrive after extremely aversive events? Am Psychol.2004;59:20. https://doi. org/10.1037/0003-066X.59.1.20 
27. Bonanno GA, Ho SM, Chan JC, Kwong RS, Cheung CK, Wong $\mathrm{CP}$, et al. Psychological resilience and dysfunction among hospitalized survivors of the SARS epidemic in Hong Kong: a latent class approach. Health Psychol.2008;27:659. https://doi. org/10.1037/0278-6133.27.5.659

28. Lee Y-R, Lee J-Y, Kim J-M, Shin I-S, Yoon J-S, Kim S-WJPi. A comparative study of burnout, stress, and resilience among emotional workers. Psychiatry Investig.2019;16:686. https:// doi.org/10.30773/pi.2019.07.10

29. Dobson H, Malpas CB, Burrell AJ, Gurvich C, Chen L, Kulkarni Jet, et al. Burnout and psychological distress amongst Australian healthcare workers during the COVID-19 pandemic. Australas Psychiatry. 2021;29:26-30. https://doi. org/10.1177/1039856220965045

30. Spielberger CD. Theory and research on anxiety. Anxiety and behavior, 1966; 1:3.

31. Samios C, Lisa MA, Amber KR. "The protective role of compassion satisfaction for therapists who work with sexual violence survivors: An application of the broaden-and-build theory of positive emotions." Anxiety Stress Coping. 2013;26:610-623. https://doi.org/10.1080/10615806.2013.784278 